acts as a physical barrier to irritants and has antibacterial activity. Encouraging reports of topical sucralfate effect on epithelialization of wounds along with its bacteriostatic property have led us to carry out a trial to evaluate its role as a topical agent in the treatment of diaper dermatitis and compare its efficacy with topical zinc oxide.

Methods A double blind randomized clinical trial was conducted from April 2008 to september 2009. Sucralfate and zinc oxide were formulated as $20 \%$ ointment with same excipients. All patients were randomly treated topically with eihter sucralfate $(\mathrm{N}=25)$ or zinc oxide $(\mathrm{N}=21)$ for 7 days. Diaper severity were obtained before treatment and days 3, 5, 7 by authors.

Results A total of 46 infants (54.3\% femate and $45.7 \%$ male) entered the study. They had a mean age 4.4+/-6.5 months. The mean age, sex, frequency of diaper changing (per day) and severity of diaper rash had no statistically significant difference between two groups. Sucralfate $20 \%$ ointment was significantly superior in healing dermatitis at days $5,7(\mathrm{p}<0.05$ and 0.01 respectively and had significant shorter healing time (3.24+/-2.02 days) in comparison with zinc oxide $20 \%$ ointment (5.42+/- 2.39 days) (pvalue $=0.002$ ).

Conclusion Since sucralfete in topical formulations acts as a phsical barrier with proved safety and has no noticeble absorption it may become a potential treatment for diaper dermatitis.

\section{THE EFFECTS OF ENVIRONMENTAL TOBACCO SMOKE ON PNEUMONIA RISK IN CHILDREN UNDER 7YEARS IN NORTHERN NIGERIA}

doi:10.1136/archdischild-2012-302724.0381

E Odiase, Children/Adolescents as SmokeFree ExamplesCASE. Epidemiology, University of Ibadan, SmokeFree Foundation, Abuja, Nigeria

Background The numerous adverse effects of Environmental Tobacco Smoke (ETS) on the non-smoking public have being evidenced through decades of research. This does not only affect adults but children. ETS effects on children have shown to be grave as it worsens asthma conditions, increases pneumonia cases and causes Sudden Infant Death Syndrome (SIDS).

Methods Most residents in all 44 Local Government Areas (LGAs) in Kano State of Northern Nigeria took part in a population-based large-scale cross-sectional survey in Kano state from 2007-2010. Demographic information coupled with socioeconomic status, smoking status and house environment of each household member, was collected from participants.

Results Out of a total of 528, 800 people resident in 102,334 homes indentified in the survey areas and visible/present as at the time of the study, 52,888 (10\%) were children aged 7 years and below. While the prevalence of ETS exposure on children was $81 \%$, the prevalence of reported pneumonia cases was 3.5\%. Multiple logistic regression analysis showed that exposure to ETS was independently associated with reports of pneumonia cases (adjusted odds ratio 1.55, 95\% CI 1.25 to 1.92). The prevalence of tobacco smoking was higher among men than women (63.5\% vs $44.1 \%)$. It is estimated that $32.7 \%$ of childhood pneumonia in the northern region of Nigeria is attributable to ETS.

Conclusions Attention should be given to reduction to children's exposure to ETS not only in Nigeria but in all affected areas mostly all parts of the world.

\section{IMPROVING ASTHMA MANAGEMENT FOR SOUTH ASIAN CHILDREN; WHOSE PRIORITIES MATTER?}

doi:10.1136/archdischild-2012-302724.0382

'M Lakhanpaul, ${ }^{2} \mathrm{D}$ Bird, ${ }^{3} \mathrm{~L}$ Culley, Management and Interventions for Asthma (MIA) Research Team. 'General and Adolescent Unit, University College London, Institute of Child Health, London; ${ }^{2 M e d i c a l}$ and Social Care Education, University of Leicester; ${ }^{3}$ Health and Life Sciences, De Montfort University, Leicester, UK
Background South Asian Children present more frequently than White British Children to emergency departments with acute asthma: tailored interventions may redress this. The Management Interventions for Asthma project (MIA) assessed the feasibility of involving adults, parents and children from this potentially 'hard to reach' ethnic community alongside healthcare professionals (HCPs) in prioritising components for tailored asthma interventions.

Methods Eleven issues identified as barriers to asthma management following interviews with community members, parents, children and HCPs were presented at community based events by the MIA team utilising interpreters/facilitators for simultaneous 5 -way translation. HCPs were sent information electronically.

Forty six community members, 22 parents, 19 children and 13 HCPs used Borda ranking to prioritise the issues for subsequent development of interventions in the current health care system.

Results Getting a diagnosis was ranked first by parents and community members but last by HCPs. Language barriers were ranked first by HCPs. Children prioritised managing acute asthma attacks. Conclusions Ethnicity and language need not be barriers to involving South Asian families in health services research.

It is crucial to include community members, families and children in the development of tailored interventions as well as HCPs. Relying on HCPs alone could lead to key issues being missed or priorities misjudged.

Acknowledgement This project was funded by the NIHR Health Services and Delivery Research programme (ref 09/2001/19). The views and opinions expressed therein are those of the authors and do not necessarily reflect those of the HS\&DR programme, NIHR, NHS or the Department of Health

\section{A RAPID BLOOD NGAL ASSAY FOR DETECTION OF RENAL CORTICAL DEFECT IN INFANTS WITH FEBRILE UTI: A PROSPECTIVE STUDY}

doi:10.1136/archdischild-2012-302724.0383

SW Nam, MK Kim, WH Seo, YJ Rhie, HE Yim, BM Choi, KH Yoo. Department of Pediatrics, Korea University College of Medicine, Ansan-Si, Republic of Korea

Background and Aims Infants with renal cortical defect due to acute pyelonephritis (APN) may be associated with an increased risk of progressive kidney damage. Neutrophil gelatinase-associated lipocalin (NGAL) is produced and secreted by renal tubule cells at low levels, but the amount produced and secreted increases dramatically after ischemic, septic, or nephrotoxic injury of the kidneys.

To investigate the usefulness of a rapid blood NGAL assay as a diagnostic marker of cortical defect in infant with febrile UTI at the bedside.

Methods Sixty-three infants with suspected febrile UTI were divided into a documented UTI group $(n=49)$ and a non UTI group $(n=14)$. UTI group were divided into cortical defect (UTI-D) group $(n=26)$ and non cortical defect (UTI-N) group $(\mathrm{n}=23)$ according to the result of DMSA scan. Blood NGAL concentrations were analyzed using a commercial kit (Triage NGAL test) by fluorescence immunoassay.

Results NGAL concentrations were significantly higher in UTI-D group $(68.0[60.0-172.5] \mathrm{mg} / \mathrm{mL})$ than in UTI-N group $(60.0[60.0$ $86.5] \mathrm{mg} / \mathrm{mL})$ and in non UTI group $(60.0[60.0-60.0] \mathrm{mg} / \mathrm{mL})$. In UTI-D groupin, NGAL concentrations were significantly decreased after antibiotic therapy $(60.0[60.0-71.2] \mathrm{mg} / \mathrm{mL})$. The area under the ROC curve of NGAL for the detection of cortical defect was 0.74 $(p=0.004)$. The best cutoff NGAL concentrations for the detection of cortical defect was found to be $61.5 \mathrm{mg} / \mathrm{mL}$ (sensitivity: $69.2 \%$; specificity $78.2 \%$ ).

Conclusions Although it is not a stand-alone test, the rapid determination of blood NGAL concentration provides valuable information quickly, concerning the distinction between APN and lower UTI, for determining the clinical course of infant with febrile UTI. 